\title{
Naturwaldreservate: welche, wo und wofür? (Essay)
}

Kurt Bollmann

Jörg Müller
Eidgenössische Forschungsanstalt für Wald, Schnee und Landschaft $(\mathrm{CH})^{*}$

Nationalpark Bayerischer Wald (DE)

\begin{abstract}
Natural forest reserves: selection criteria, where and what for? (Essay)
The question "How large should the total extent of strict natural forest reserves be?" dominates the current debate about the need of unmanaged forests for biodiversity conservation in Central Europe. However, within a system of close-to-nature forest management, the quality, location, composition and distribution of natural forest reserves might have higher impacts on the diversity of species, communities and natural processes than the reserves' extent alone. Strictly speaking, the correct answer about the minimal required surface is directly related to the superior conservation objectives. In addition, the required ratio of forest reserves in relation to the total forest area is influenced by other factors such as the abundance and distribution of forests pirmarily managed for conservation objective, protected forest biotopes and old-growth stands as well as the general standards for an integrative, close-to-nature silviculture. Since concrete, superior objectives for forest biodiversity conservation are still missing in Central Europe, we put the focus of this article on the criteria that influence the conservation-specific impact and quality of strict natural forest reserves. These are amongst others the extent and compactness of a reserve, its habitat continuity and connectivity, the representativeness of forest types, their species composition and biogeographic distribution, a reserve's site heterogeneity and naturalness of its vegetation as well as the abundance of key structures and target species.
\end{abstract}

Keywords: biodiversity, nature protection, forest reserves, natural dynamics, selection criteria doi: $10.3188 /$ szf.2012.0187

*Zürcherstrasse 111, CH-8903 Birmensdorf, E-Mail kurt.bollmann@wsl.ch

Z ahlreiche Studien belegen die Bedeutung von ungenutzten gegenüber genutzten Wäldern für anspruchsvolle Waldorganismen (Winter et al 2005, Müller et al 2007a, Müller et al 2007b, Müller et al 2008b). Auch wenn dies nicht für alle Artengruppen in gleichem Masse zutrifft (Paillet et al 2010), ist die Notwendigkeit von Naturwaldreservaten beziehungsweise unbewirtschafteten Wäldern zur Erhaltung zahlreicher gefährdeter Arten der Alters- und Zerfallsphasen sowie zur Förderung vielfältiger natürlicher Prozesse im Wald fachlich unbestritten. Der nationale Anteil an Naturwaldreservaten (auch Totalreservate genannt) an der ganzen Waldfläche variiert in Europa zwischen 0.0002 und 6.6\% (Bücking 2007), mit Werten von 2.5, 0.29 und $0.22 \%$ für die Schweiz (Bolliger et al 2012, dieses Heft), Deutschland (Meyer et al 2007) beziehungsweise Österreich (Frank et al 2010). Daneben wurde gemäss dem dritten Landesforstinventar auf 18\% der Schweizer Waldfläche seit mindestens 50 Jahren kein Holz mehr genutzt (Duc et al 2010). Diese Wälder haben zwar nicht den Status von geschützten Naturwaldreservaten, unterstützen den Prozessschutz aber trotzdem. Die staatlichen Zielgrössen für vertraglich gesicherte Naturwaldreservate liegen in der Schweiz und in Deutschland bei je 5\% (BMU 2007, FDK \& Buwal 2001'), in Österreich bei 0.17\% (10000 ha; Frank et al 2010). Die von Naturschutzorganisationen geforderten Werte liegen zwischen 5 und 10\% (Broggi \& Willi 1993, Scherzinger 1996, Indermühle et al 1998, Pro Natura 2011, SVS 2011). Der Flächenanteil für Natur- und Sonderwaldreservate zusammen ist in der Regel doppelt so gross. Diese Werte wurden ursprünglich über Annäherungsverfahren bestimmt, wobei vor allem Arten-Areal-Beziehungen, populationsbiologische Erfordernisse und Minimumareale für ein räumliches Mosaik von verschiedenen Waldentwicklungsphasen berücksichtigt worden sind.

Wir werden in diesem Artikel keine neue Flächenquote für Naturwaldreservate in Mitteleuropa

\footnotetext{
1 FDK, BUWAL (2001) Leitsätze einer Waldreservatspolitik Schweiz. Bern: Bundesamt Umwelt Wald Landschaft. 6 p www.news.admin.ch/NSBSubscriber/message/attachments/ 4422.pdf (27.3.2012)
} 
Bäume, Biotopbäume, Totholz, Substratvielfalt, überdurchschnittlich viele Grenzlinien (Ökotone), geschlossene Stoffkreisläufe und ein abwechslungsreiches mikrotopografisches Relief sowie ein graduell abgestuftes Licht- und Wärmeregime.

Die vielfältigen Strukturen und Substrate schaffen eine Mannigfaltigkeit an ökologischen Nischen, die zahlreichen anspruchsvollen, seltenen und gefährdeten Arten einen Lebensraum bieten, den sie im bewirtschafteten Wald nicht ausreichend vorfinden. $\mathrm{Zu}$ ihnen gehören Moose, Flechten, Schnecken, Pilze und verschiedene Vogel- und Fledermausarten, vor allem aber die auf Totholz spezialisierten Insektenarten (Bütler \& Lachat 2009). Es wird geschätzt, dass 20-30\% der Waldfauna direkt oder indirekt von Totholz lebt (Siitonen 2001). Zahlreiche Studien haben einen positiven Zusammenhang zwischen dem Totholzvolumen und der Vielfalt von saproxylisch lebenden Organismen nachgewiesen (z.B. Martikainen et al 1999, Schiegg 2000a). Allerdings ist in Mitteleuropa - im Unterschied zu Skandinavien (Siitonen \& Saaristo 2000) - keine Art bekannt, die nur in Naturwäldern vorkommt. Die Chance, einen Mulmhöhlenbewohner wie den Kleinen Ameisenkäfer (Euthiconus conicicollis) in einem nicht bewirtschafteten Wald zu finden, ist zwar grösser als im bewirtschafteten Wald. Aber auch Kulturwaldformen wie Mittelwald, Waldweide, Kastanienselven oder Parkanlagen und Alleen können seltene Mikrohabitate aufweisen. Weil aber die Habitatkontinuität in nicht geschützten Wäldern längerfristig nicht garantiert werden kann, bieten Naturwaldreservate den besten Schutz auch in Zeiten hoher Holzpreise oder eines grossen Energieholzbedarfs. Seltene Pilze wie die Zitronengelbe Tramete (Antrodiella citrinella), die aufgrund des heutigen Wissens auf minimale Totholzvolumen von $120 \mathrm{~m}^{3}$ /ha angewiesen sind, können in der mitteleuropäischen Kulturlandschaft längerfristig nur in Naturwaldreservaten überleben, in denen sich urwaldähnliche Totholzvorräte ansammeln (Bässler \& Müller 2010). Wir vermuten, dass Alt- und Totholzspezialisten in solchen Habitaten zu guten Populationsbeständen heranwachsen (z.B. Schiegg 2000b, Duelli \& Wermelinger 2010). Wie weit sie von dort aus Altholzinseln in bewirtschafteten Beständen besiedeln können, hängt vom Ausbreitungspotenzial dieser Arten ab (Ranius 2006).

\section{Naturwaldreservate: eines von mehreren Naturschutzinstrumenten im Wald}

Naturwaldreservate sind keine Alleskönner oder Multifunktionstools des Naturschutzes! Man muss beachten, dass die meisten Waldbestände bei uns im Alter von 80 bis 120 Jahren noch weit davon entfernt sind, bei einer Nutzungsaufgabe die Funk- tion eines Naturwaldes zu übernehmen (z.B. Heiri et al 2009). Vorerst werden die stehende Biomasse und die Beschattung zunehmen und die Gehölzartenvielfalt abnehmen (Heiri et al 2011). Tritt keine äussere Störung ein, so sind solche Wälder mindestens in den nächsten 80 bis 100 Jahren naturschutzfachlich wenig interessant. Obwohl sich das Struktur- und Totholzangebot drei bis vier Jahrzehnte nach Bewirtschaftungsaufgabe wesentlich erhöht (Bütler \& Lachat 2009), zeigen Untersuchungen von Moning \& Müller (2009) und Moning et al (2010), dass Wälder der montanen Stufe erst ab einem Bestandesalter von 200 Jahren seltenen Arten einen Lebensraum bieten. Objektiv betrachtet investieren wir also heute mit zahlreichen Naturwaldreservatsausweisungen in eine ökologische Leistung, die erst in einer Zeit erbracht wird, wenn die aktuell laufenden Reservatsverträge abgelaufen sind. Diese Aussage gilt in erster Linie für laubwalddominierte Waldtypen. In Wäldern, die natürlicherweise stärkeren Störungen wie Überflutung (Auenwälder) oder Insektenfrass (hochmontane Fichtenwälder) unterliegen, können sich naturnahe Strukturen bereits nach kurzer Zeit ausbilden.

Es wäre wichtig, sich in der Debatte um Naturwaldreservate an einer übergeordneten Zielsetzung für den Waldnaturschutz in Mitteleuropa orientieren zu können und die gesellschaftlich akzeptierten Anteile der Waldfläche für Waldreservate zielgerichtet auf die Reservatstypen Natur- und Sonderwaldreservate aufzuteilen. Diese Reservatstypen sind in ihrer Wirkung komplementär (Bollmann 2011). Sonderwaldreservate sind effektive Instrumente, um mit gestaltenden forstlichen Massnahmen eine Entwicklung einzuleiten und auf ein Ziel hinzulenken. Die Entwicklung von Naturwaldreservaten ist ergebnisoffen und wird massgebend beeinflusst durch natürliche Störungen. Dies ist mit ein Grund, warum Sonderwaldreservate als Instrumente zur dringlichen Förderung von gefährdeten Arten effektiver sein können als Naturwaldreservate (z.B. Bollmann et al 2008, Ehrbar et al 2011). Dies trifft insbesondere auf viele gefährdete Gefässpflanzen und Tagfalter zu, die auf nährstoffarme, trockene oder nasse Waldbestände mit einem guten Lichtangebot angewiesen sind (Schiess \& Schiess-Bühler 1997, Indermühle et al 1998).

Weiter gilt es auch zu beachten, dass sich Naturwaldreservate in Mitteleuropa nicht dazu eignen, überlebensfähigen Populationen (mindestens 500 Individuen; Lande \& Barrowclough 1987) von Arten mit grossen Streifgebieten wie Luchs oder Weissrückenspecht genügend Lebensraum zu bieten. Je nach Ernährungstyp und Mobilität solcher Arten kann der Raumbedarf mehrere Hundert bis tausend Quadratkilometer betragen (Scherzinger 1996). Das Minimumareal solcher Arten ist also kein nützliches Kriterium, um die erforderliche Fläche von Natur- 
Abb 2 Die Schweiz hat eine besondere Verantwortung für die Erhaltung der Arvenwälder. Foto: Sabine Brodbeck

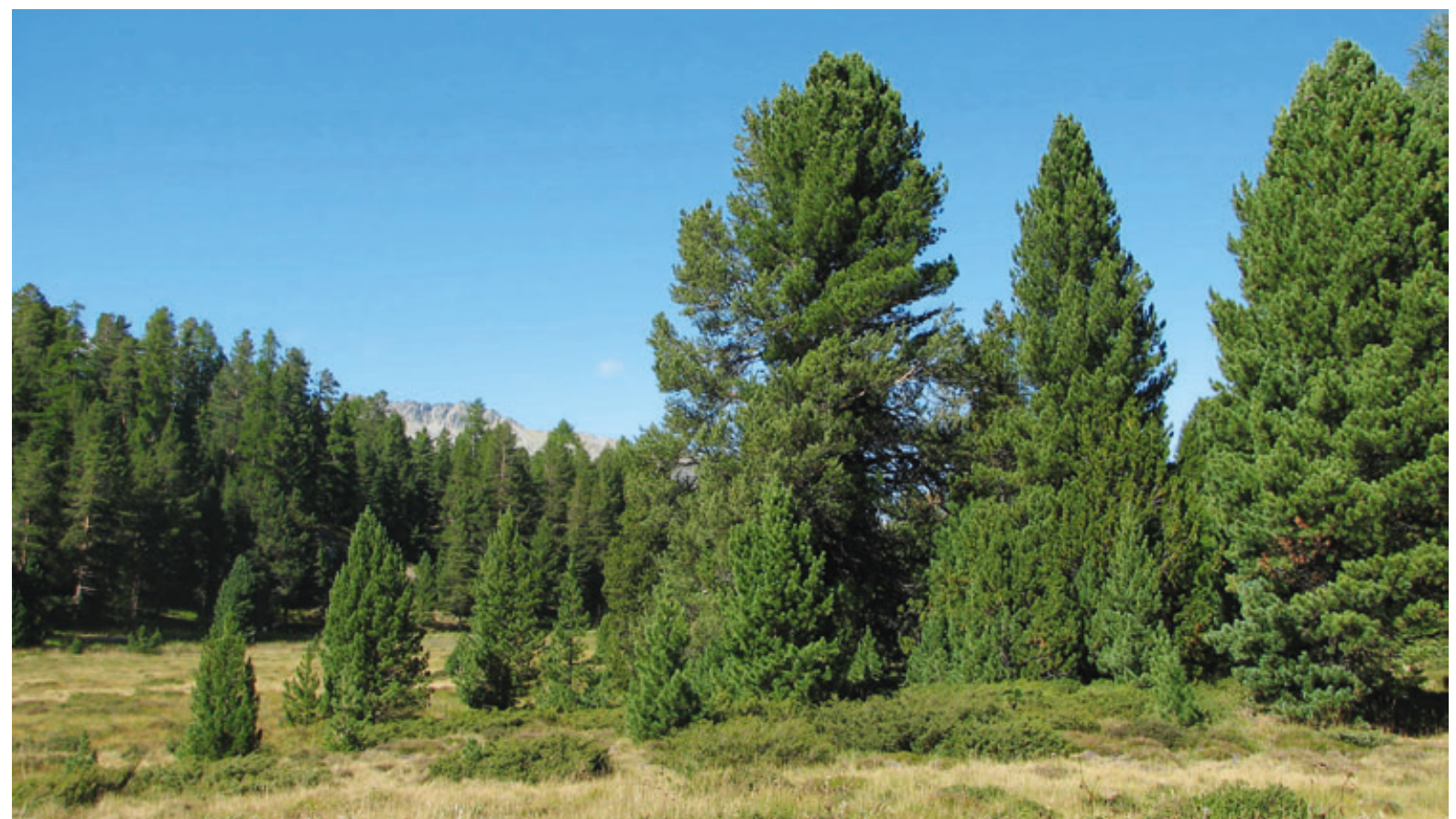

waldreservaten in Kulturlandschaften zu ermitteln. Das Kriterium ist eigentlich nur in einem Waldwirtschaftssystem mit vollständiger Trennung von Schutz und Nutzung relevant. Aus Siedlungsdichten des Weissrückenspechts von 0.7 bis 1.38 Brutpaaren pro km² im Alpenraum (Frank 2002; Bühler, schriftliche Mitteilung) errechnet sich theoretisch ein minimaler Flächenbedarf von 180 bis $350 \mathrm{~km}^{2}$ Lebensraum mit totholzreichen Laubholzbeständen (> $100 \mathrm{~m}^{3}$ Totholz pro ha) für 250 Brutpaare. Diese Anforderungen machen deutlich, dass solche Ziele nur in einem vernetzten System mit Naturwaldreservaten und integrativen Artenschutzmassnahmen erreicht werden können (Bühler 2009).

\section{Standörtlich repräsentative, naturnahe und funktionelle Natur- waldreservate}

In der aktuellen Diskussion über Naturwaldreservate in Mitteleuropa wird die Frage des minimal notwendigen Waldflächenanteils sehr hoch gewichtet. Um sie zu beantworten, müsste man die übergeordneten Ziele für den Schutz der Biodiversität im Wald im Zusammenhang mit den nationalen Biodiversitätsstrategien kennen (Bollmann et al 2009). Entsprechende Diskussionen finden zurzeit statt, politisch konsolidierte Ziele fehlen aber weitgehend. Weil der Prozess der Festlegung von Naturwaldreservaten in der Schweiz, Deutschland und Österreich schon läuft, gewichten wir den Aspekt der Lebensraumqualität der Naturwaldreservate höher als jenen ihrer Fläche pro Land oder Region. Diese Qualität kann mit einer guten Kombination von Kriterien bei der Planung und Ausscheidung von Naturwaldreservaten optimiert werden.

\section{Grösse und Kompaktheit}

Zunehmende Fläche bringt standörtliche Vielfalt und ökologische Resilienz. Eine Verzehnfachung der Reservatsfläche bewirkt in der Regel eine Verdoppelung der Artenzahl. Allerdings hängt die Bedeutung der Reservatsgrösse auch von der Artengruppe sowie der Matrix und der Vernetzung der Reservate ab. Je besser die Lebensraumqualität auf der ganzen Waldfläche ist und je besser der Austausch von seltenen und gefährdeten Arten zwischen Naturwaldreservaten funktioniert, desto geringer ist deren Flächenbedarf. Lange war man der Meinung, ein Naturwaldreservat müsse im Flachland mindestens 50 und im Gebirge 100 ha gross sein. Diese Minimalflächen wurden vom Phasenzyklus und Sukzessionsmosaik für verschiedene Waldtypen hergeleitet (Albrecht 1991). Aus Sicht der Biodiversitätsforschung lassen sich diese Referenzgrössen aber nicht bestätigen. Für Arten mit grossen Raumansprüchen wie Auerhuhn oder Schwarzstorch sind sie ohnehin viel zu klein. Für Arthropoden können bereits 3 bis 5 ha ausreichen, um eine Population langfristig zu erhalten (Müller 2005). Zudem haben azonale Waldgesellschaften auf Sonderstandorten wie Bruch-, Schlucht- und Blockschuttwälder nur selten eine grössere Ausdehnung. Man muss aber beachten, dass die ökologische Resilienz mit der Flächengrösse steigt, weil die Wahrscheinlichkeit des Aussterbens einzelner Arten mit zunehmender Populationsgrösse abnimmt. Für grossflächig dynamische Prozesse wie Borkenkäfergradationen, Überschwemmungen, Windwürfe oder Feuer sollten die Reservatsflächen deutlich grösser sein als ein paar Hektaren. Im über 10000 ha grossen Nationalpark Bayerischer Wald wurden Waldbestände auf knapp 50\% der Fläche durch die Massenvermehrung des Borkenkäfers zum Absterben gebracht. Damit ist auch am Ende einer 


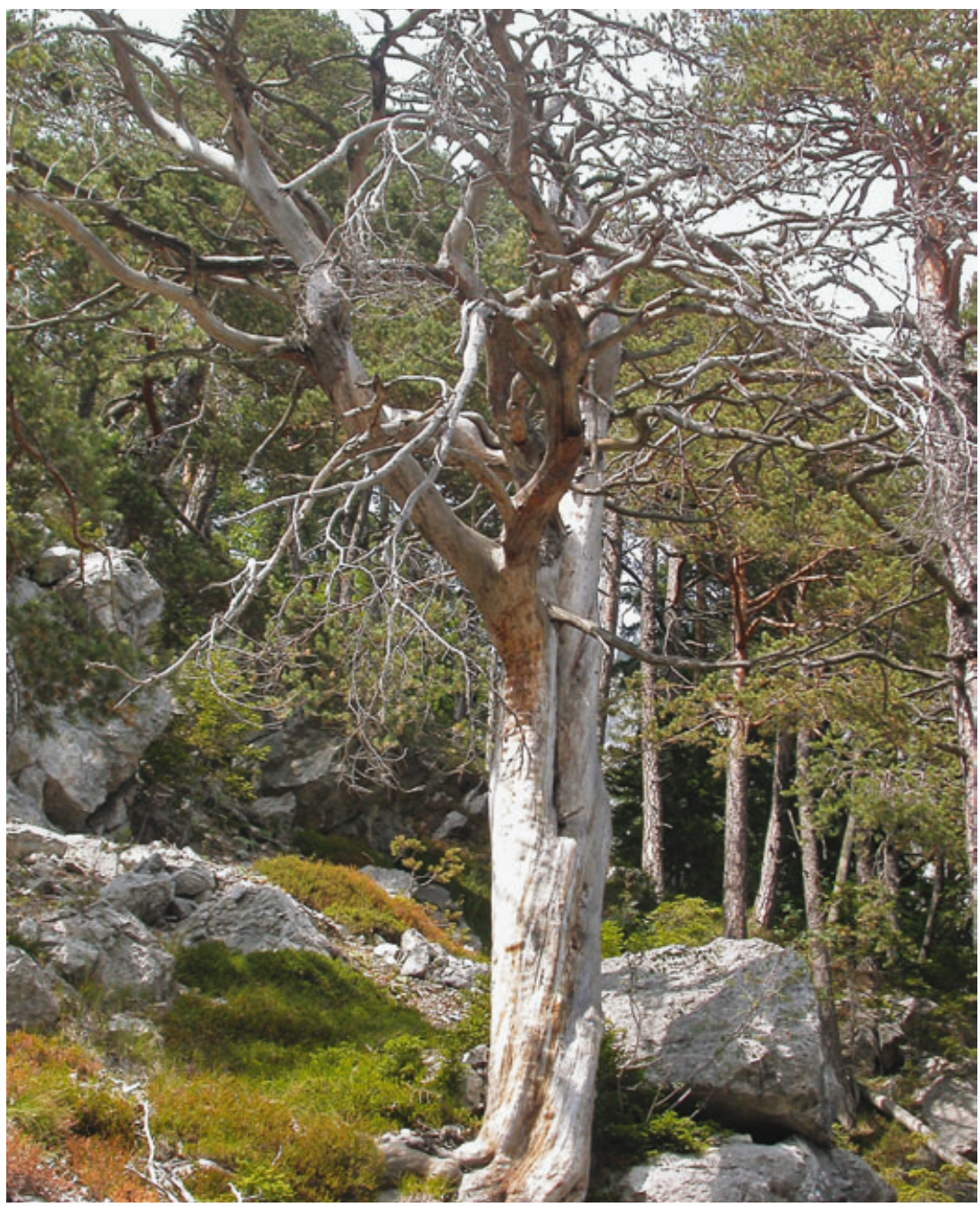

Abb 3 Standörtlich heterogene Wälder bieten eine hohe Nischenvielfalt für die Entwicklung von artenreichen Beziehungsnetzen in Waldökosystemen. Foto: Andrea Jazarbek-Müller

solchen Gradation im Schutzgebiet der ganze Gradient von dichten zu lichten und zerfallenen Waldbeständen vorhanden (Müller et al 2010b). Das Waldreservat Rorwald im Kanton Obwalden hat eine Grösse von 200 ha und umfasst seit dem Orkan Lothar ausgedehnte Sturmflächen in den Fichten- und Fichten-Tannen-Wäldern neben Moorrand-Föhrenwäldern im Klimaxstadium (Reich et al 2010; Abbildung 1). Obwohl der Borkenkäfer in der 70 ha grossen Kernzone die grosse Mehrheit der Fichten und Bergföhren zum Absterben gebracht hat, ist im gesamten Reservat noch ein breites Spektrum an standorttypischen Entwicklungsphasen und Waldformen vorhanden. Diese Beispiele von natürlichen Bestandesmosaiken dank grossflächigen Störungen sind ein gutes Argument für Naturwaldreservate von mehreren Hundert bis tausend Hektaren. In solchen Reservaten können sämtliche Sukzessionsphasen auch nach grösseren natürlichen Störungen nebeneinander vorkommen, wodurch den Habitatspezialisten stets alternative Lebensräume in erreichbarer Distanz zur Verfügung stehen. Dies gilt vor allem für Waldtypen mit relativ grossflächiger natürlicher Dynamik wie die Fichtenwälder, im Gegensatz zu den Buchenwäldern mit ihrer eher kleinräumigen
Dynamik. Es gilt aber auch zu beachten, dass kleine Reservate meistens eingebettet sind in grössere Waldgebiete und sich viele Arten über umgebende Waldbestände ausbreiten können (Müller \& Gossner 2007). Die Kompaktheit dürfte aber gerade bei kleinen Waldreservaten ein wichtiges Qualitätsmerkmal sein, weil dadurch unerwünschte Randeffekte (Fenger 1996) verringert werden.

\section{Internationale Verantwortung}

Als Verantwortungsarten werden Arten bezeichnet, welche in einer Region im Vergleich zur internationalen Situation in überdurchschnittlich hohen Beständen vorkommen, unabhängig vom Gefährdungsgrad (Keller \& Bollmann 2001). Analog kann für Pflanzengesellschaften das Kriterium der Totalfläche verwendet werden, um die internationale Verantwortung herzuleiten. Deutschland hat beispielsweise eine besondere Verantwortung für die Erhaltung der Buchenwälder (Walentowski et al 2010). In der Schweiz sind es die Bergföhrenwälder, Arvenwälder (Abbildung 2), eibenreiche Buchenwälder und subalpine Auenwälder (Indermühle et al 1998).

\section{Standörtliche Vielfalt, natürliche Vegetation} und Abundanz von Schlüsselstrukturen

Standörtliche Vielfalt schafft unterschiedliche Nischen und Konkurrenzverhältnisse und ist die Voraussetzung für die Entwicklung verschiedener Vegetationseinheiten (Abbildung 3). Dadurch kommt es zum syntopen Vorkommen von Arten mit verschiedenen Habitatansprüchen und Verbreitungsschwerpunkten in Europa. Die mitteleuropäischen Gebirgsregionen bieten beste Voraussetzungen, um geologische, orografische oder klimatische Unterschiede und Vielfalt bei der Planung von Naturwaldreservaten zu berücksichtigen. Weiter soll darauf geachtet werden, dass die aktuelle Baumartenzusammensetzung möglichst der potenziell natürlichen Vegetation entspricht (Indermühle et al 1998, Frank 2009, Meyer et al 2011). Aber auch das Vorkommen von Schlüsselstrukturen wie Baumhöhlen kann die Diversität von Lebensgemeinschaften entscheidend beeinflussen (Carlson et al 1998). So kommt es bei Spechthöhlen zu Folgenutzungen durch andere höhlenbrütende Vogelarten (Aitken et al 2002), darunter Hohltaube, Dohle und Raufusskauz, aber auch Bilche, Fledermäuse und Insekten (Abbildung 4). Ausführlich wurde dies auch für Mikrohabitatstrukturen an einzelnen Bäumen gezeigt, die in ungenutzten Altbeständen häufiger und in vielfältigeren Formen vorkamen als in genutzten Beständen, die gleichzeitig deutlich ärmer an gefährdeten Käferarten waren (Winter \& Möller 2008).

\section{Habitatkontinuität}

Je weiter die Tradition an naturnahen Strukturen in einem Wald zurückreicht, desto höher ist die 


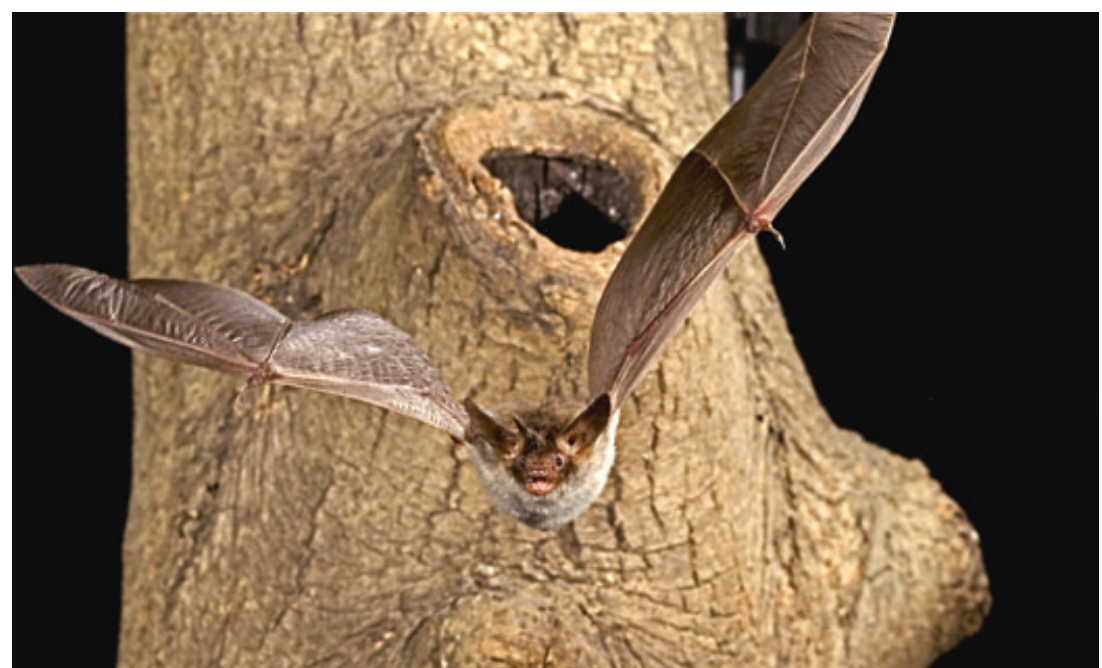

Abb 4 Die Bechsteinfledermaus (Myotis bechsteini), die selber keine Höhlen zimmert, ist auf die gestaltende Vorleistung von Spechten oder auf natürliche Astlöcher angewiesen. Foto: René Janssen gen Substrat- und Nischenangebot. Deshalb haben die Zeit seit der letzten Nutzung und die Wahrscheinlichkeit des Auftretens von natürlichen Störungen entscheidenden Einfluss auf das Entwicklungspotenzial von Naturwaldreservaten. Dieser Umstand erschwert die Planung von Naturwaldreservaten in gut erschlossenen Tieflandgebieten, wo biologisch alte Wälder sehr selten sind. Auswertungen des dritten Landesforstinventars (Cioldi et al 2010) zeigen, dass Waldbestände mit einem Alter über 180 Jahren im Schweizer Mittelland einen Flächenanteil von weniger als einem Prozent umfassen, während sie in den Alpen je nach Region 4 bis 9\% ausmachen.

\section{Standorte mit hoher oder geringer natürlicher Dynamik}

Solche Standorte sind fast ausnahmslos Lebensräume mit einer hohen Vielfalt an seltenenoder spezialisierten Arten. Typische Vertreter für Standorte mit hoher Dynamik sind die Auen- und Feuchtwälder, die zu den gefährdetsten Waldtypen Mitteleuropas gehören Es können auch Felssturzgebiete sein, die Reliktarten aus der Wärmezeit wie dem eng an lückige Waldföhrenbestände gebundenen Prachtkäfer der Gattung Buprestis (z.B. B. splendens, $B$. novemmaculata) einen Lebensraum bieten. Untersuchungen auf ehemaligen und rezenten Waldbrandflächen zeigen, dass Arten wie der Erdbeerspinat (Blitum virgatum) oder auf Brandstellen angewiesene Lauf- und Prachtkäfer plötzlich wieder eine Nische haben (Moretti et al 2010, Pradella et al 2010, Wohlgemuth et al 2010a). In Windwurfflächen liessen sich hohe Abundanzen an Rote-Liste-Arten und Totholzspezialisten nachweisen (Wermelinger et al 1995, Bouget \& Duelli 2004). Ähnliche Ergebnisse lieferten Berg et al (1994), die zeigten, dass in Schweden 20\% der Käferarten der Roten Liste von grossen Sturmwurfflächen profitieren, während nur 4\% dadurch benachteiligt werden. Totholzinseln, die auf die Aktivität von Borkenkäfern zurückgehen, hatten eine positive Wirkung auf die Insektenvielfalt im Nationalpark Bayerischer Wald (Müller et al 2008a). Auch die Bedeutung von extremen Überschwemmungen für unterschiedliche Artengruppen wurde in einer Studie nach dem Elbehochwasser von 2002 dokumentiert (Ilg et al 2008).

Standorte mit geringer natürlicher Dynamik zeichnen sich durch ein stark limitiertes Angebot an Nährstoffen oder Wasser aus. Hier können sich unter guten Lichtverhältnissen seltene Artengemeinschaften entwickeln, die auf wüchsigen Standorten von dominanten Gehölzarten verdrängt werden. Beispiele dafür sind Flaumeichenwälder und Moorrand-Föhrenwälder.

\footnotetext{
3 SENN-IRLET B (IN PRESS) Phellinus laricis - Goldrandiger Feuerschwamm. Birmensdorf: Eidgenöss Forsch.anstalt WSL, Merkblätter Arten. 2 p.
} 


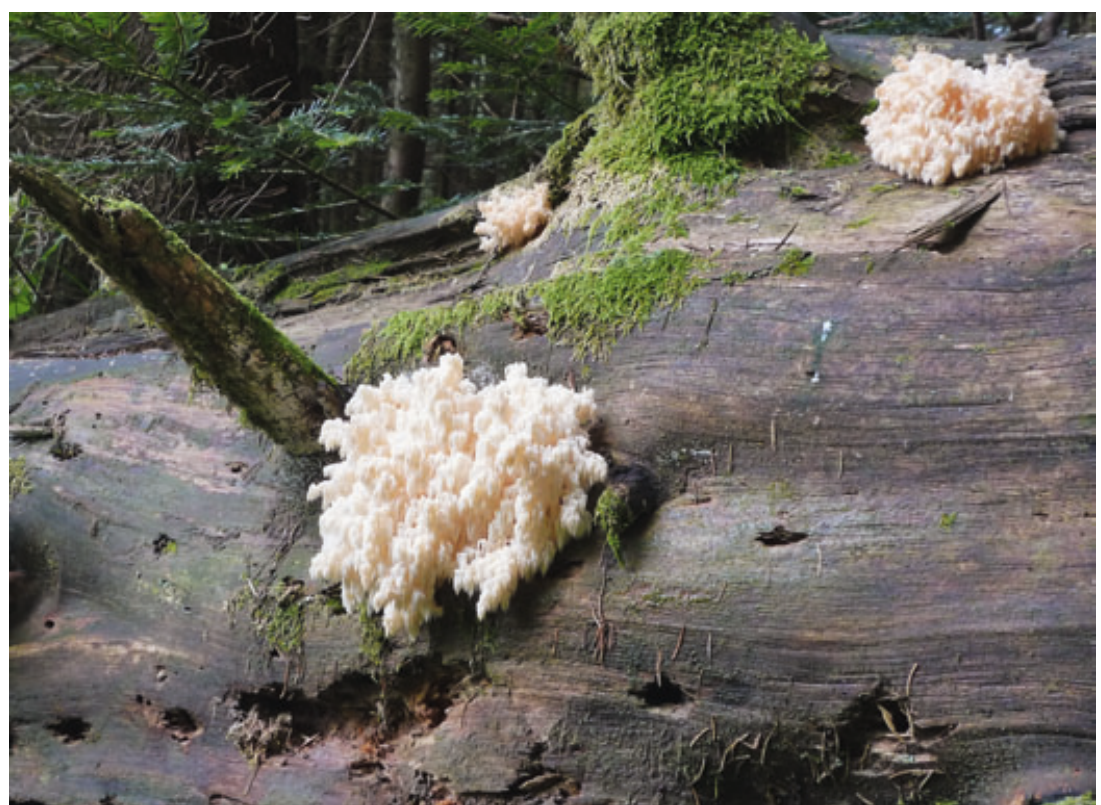

Abb 5 Viele Moos-, Flechten- und Pilzarten sind sensitiv gegenüber forstlicher Nutzung und auf lange Entwicklungszeiten der Waldhabitate angewiesen. Im Bild der Ästige Stachelbart (Hericium coralloides), der nur auf stark zerfallenem Buchenholz vorkommt.

\section{Repräsentativität der Waldtypen und biogeografische Verteilung}

Ein weiteres Kriterium ist die repräsentative Verteilung der Naturwaldreservate über verschiedene Höhen-, Klima- und Wuchszonen. Möglichst viele natürlich vorkommende Waldgesellschaften eines Landes sollten im Reservatsnetzwerk angemessen vertreten sein. Entsprechende Angaben für die Schweiz und Bayern findet man bei Indermühle et al (1998) beziehungsweise Seibert \& Hagen (1974). Die Diversität der repräsentierten standorttypischen Waldformen erweitert die Zahl der ökologischen Nischen und erhöht so die Gesamtartenzahl über alle vertretenen Waldformen (Betadiversität; Müller \& Gossner 2010, Gossner \& Müller 2011). Ein grossräumiges Reservatsnetz soll also nicht nur das aktuelle Vorkommen von bedeutenden Arten (Hotspots der Artenvielfalt) berücksichtigen, sondern zusätzlich möglichst viele biogeografische Regionen abdecken.

\section{Vernetzung}

Allgemeingültige Kriterien zur Vernetzung sind schwierig herzuleiten. Es gilt zu bedenken, dass Besiedlungen seltene Ereignisse sind, die in Untersuchungen nur ausnahmsweise erfasst werden. Jüngere Studien zeigen, dass die Empfehlungen für Vernetzungen wissenschaftlich nur selten erhärtet sind und bei der Planung von Reservatsnetzwerken eher intuitiv berücksichtigt werden müssen (Abbildung 7). Sinnvoll ist es sicher, das Netz ausgehend von bedeutenden, aktuell besiedelten Gebieten oder Hotspots zu erweitern. So haben Untersuchungen am Auerhuhn (Tetrao urogallus) gezeigt, dass die Wahrscheinlichkeit für den Austausch zwischen Populationen $\mathrm{ab}$ einer Distanz von $10 \mathrm{~km}$ stark ab-

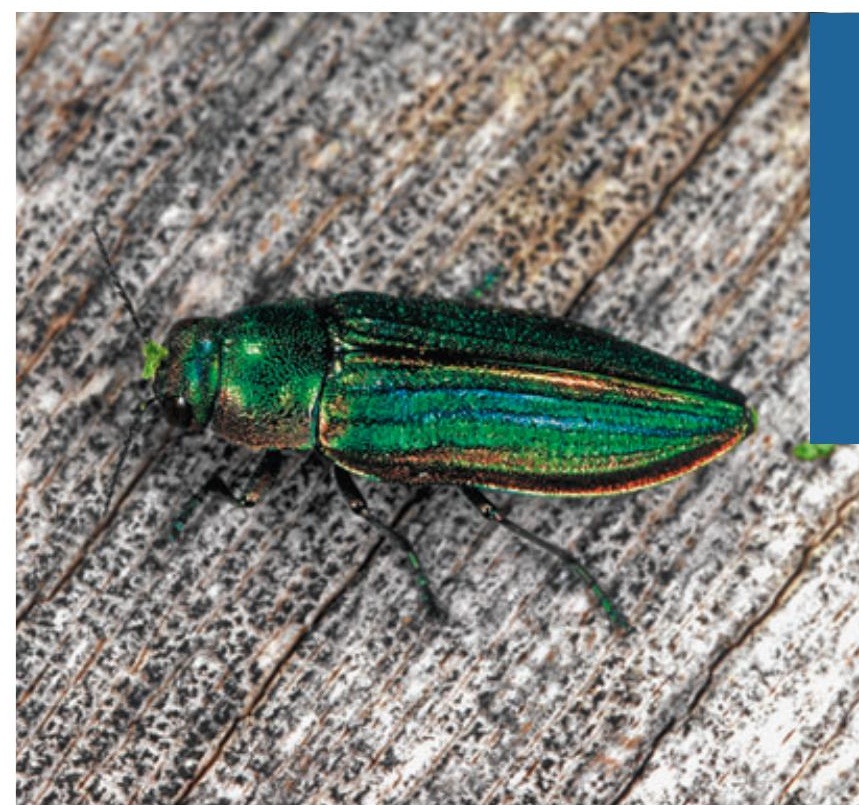

Abb 6 Waldbestände, die noch Restpopulationen von seltenen Habitatspezialisten wie zum Beispiel den Goldstreifigen Prachtkäfer (Buprestis splendens) beherbergen, eignen sich als Kandidaten für Naturwaldreservate. Foto: Heinz Bussler

nimmt (Bollmann et al 2011). Für andere, wenig mobile Arten wie Schnecken dürfte bereits ein Bruchteil dieser Distanz zu gross sein. Aber auch für flugfähige Arten wie den Wachtelweizen-Scheckenfalter (Mellicta athalia) wurde gezeigt, dass Waldlichtungen in Distanzen von $600 \mathrm{~m}$ nicht mehr kolonisiert werden (Warren \& Key 1991). Diese Beispiele unterstreichen die Bedeutung von Zielarten bei der Planung von Reservatsnetzwerken.

\section{Zielarten}

Grundsätzlich ist es möglich, für die repräsentativen Waldtypen eines Gebiets Zielarten und deren Ansprüche an die Waldfläche zu bestimmen (Abbildung 8). Dies hätte den Vorteil, dass der Flächenbedarf für überlebensfähige Populationen biologisch hergeleitet und die Zielerreichung zu einem späteren Zeitpunkt überprüft werden könnte. Solche auf Einzelarten ausgerichteten Schutzkonzepte werden öfters kritisiert, haben aber den Vorteil, dass sie für die breite Bevölkerung verständlich und politisch erklärbar sind (Bollmann et al 2002, Lorenz 2010). Darüber hinaus führen sie auch zu einer neuen Bewertung von Lebensräumen. Während Letztere bislang vor allem nach Naturnähe, Seltenheit oder Ästhetik beurteilt wurden, orientiert sich ein Zielartenkonzept an den Bedürfnissen und der Entwicklung von ausgewählten Arten und der internationalen Verantwortung, die ein Land oder eine Region für eine bestimmte Art hat. Damit rücken die Fragen nach Habitatgrösse, -ausstattung und -vernetzung ins Zentrum der Betrachtung, also Planungsgrössen, die die Populationsentwicklung direkt beeinflussen.

Evolutionsbiologisch betrachtet gäbe es gute Argumente für Naturwaldreservate, welche die Funk- 
tion von Genreservaten übernehmen (Bonfils \& Bolliger 2003). Dadurch kann das zukünftige Potenzial von standörtlich angepassten autochthonen Arten und Provenienzen unter natürlicher Selektion bewahrt werden. Dies könnte gerade in Zeiten des Klimawandels von Bedeutung sein, solange wir die physiologische Plastizität und das kompetitive Potenzial der einheimischen Gehölzarten unter veränderten Umweltbedingungen und forstwirtschaftlicher Selektion noch kaum kennen. Die wissenschaftlichen Grundlagen dazu sind aber dürftig, vor allem wenn es um räumlich explizite Grundlagen geht, die die Planung von Naturwaldreservaten unter Einschluss dieses Kriteriums ermöglichen würden.

\section{Einige Zahlen}

Die obigen Ausführungen zeigen, dass - je nach naturräumlichen Besonderheiten und regionalen Schutzzielen - das eine oder andere Kriterium höher zu bewerten ist und eine Kriterienkombination den Aspekt des integralen Biodiversitätsschutzes am besten erfüllt. Dennoch bleibt für die Umsetzung und Planung die zentrale Frage unbeantwortet, mit welchem Flächenbedarf an Naturwaldreservaten zu rechnen ist. Gemäss dem «Forest Practices Code» für British Columbia sollen Alters- und Zerfallsphasen mindestens die Hälfte der Fläche einnehmen können, auf der sie unter natürlichen Bedingungen vorkämen (Fenger 1996). Es liegen aber nur für wenige Waldgesellschaften empirische Angaben zur Dauer der Entwicklungsphasen und ihrer Flächenausdehnung vor (Frank 2009). Eine vergleichende Untersuchung von ungenutzten Waldbeständen im polnischen Nationalpark Białowieża mit

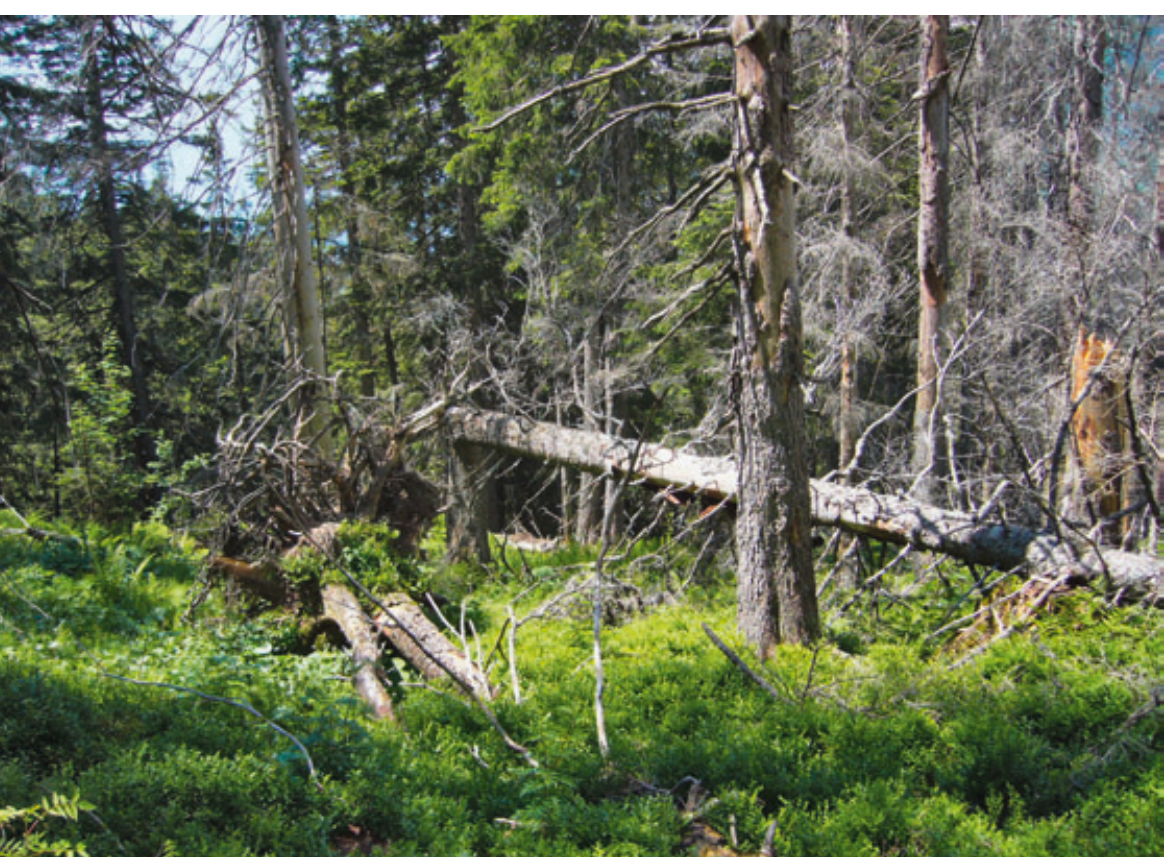

$A b b>$ Alt- und Totholzinseln tragen zur funktionellen Vernetzung der Naturwaldreservate untereinander bei.
Wirtschaftswäldern hat gezeigt, dass die Alters- und Zerfallsphasen 20 bis 35\% des Bestandsmosaiks im Eichen-Hagebuchen-Urwald ausmachen. Diese fehlen in den genutzten Wäldern (Bobiec et al 2000). Diese Zahlen liegen im Bereich der Erfahrungswerte (20-58\%) von anderen Urwäldern Europas (Müller et al 2012). Um die Nachhaltigkeit des Phasenzyklus zu garantieren, wäre bei einer vollständigen Segregation der Waldfunktionen ein minimaler Anteil an Naturwaldreservaten in diesen Grössenordnungen nötig. Eine differenzierte Waldbehandlung vermag den erforderlichen Flächenanteil für Naturwaldreservate zu reduzieren, je nach übergeordneten Schutzzielen wahrscheinlich sogar deutlich. Es muss ihr aber gelingen, einen funktionellen Verbund zwischen naturschutzfachlich wichtigen Habitaten mit Altholzinseln oder Biotopbäumen herzustellen, damit ein Austausch von saproxylobionten Käfern, Moosen, Flechten und Pilzen stattfinden kann und neu entstandene Inseln (z.B. Sturmflächen) spontan besiedelt werden können. Eine Studie im südlichen Schweden hat nachgewiesen, dass der Anteil an geeignetem Waldhabitat für Gefässpflanzen, Flechten, Moose und holzbewohnende Pilze die Dichte von Arten der Roten Liste im Umkreis von einem bis fünf Kilometern um alte Laubwälder positiv beeinflusst (Paltto et al 2006).

\section{Hindernisse auf dem Weg zum Ziel}

Im Zusammenhang mit dem Klimawandel wird immer häufiger ein räumlich flexibles Reservatsdesign gefordert, bei dem die Reservatsgrenzen an sich ändernde Bedingungen angepasst oder ganze Reservate verschoben werden können (Pressey et al 2007). Dies mag gewisse Vorteile haben, aber auch offensichtliche Nachteile. Dazu gehört der Verlust der Habitattradition, einer der wichtigsten Eigenschaften von Naturwaldreservaten. Deshalb plädieren wir für einen zurückhaltenden Umgang mit dynamischen Naturwaldreservatskonzepten. Wird das Selektionskriterium der standörtlichen Vielfalt in die Planung integriert, so sollten die Effekte des Klimawandels ohnehin geringer ausfallen, worauf Resultate von neueren Studien hinweisen (Scherrer \& Körner 2011). Darüber hinaus sind für viele Waldarten die Strukturen und das Mikroklima wichtiger als das Regionalklima. Somit können naturnahe und strukturreiche Waldbestände, wie sie in alten Naturwaldreservaten vorkommen, die Wirkung von Klimaänderungen abpuffern (Moning \& Müller 2009, Bässler et al 2010, Raabe et al 2010). Für viele Arten scheint darüber hinaus in geeignetem Mikroklima ein Überleben auch bei Klimaerwärmung möglich zu sein, was jüngst zu einer Abschwächung stark pessimistischer Aussterbeprognosen geführt hat (Hof et al 2011) 


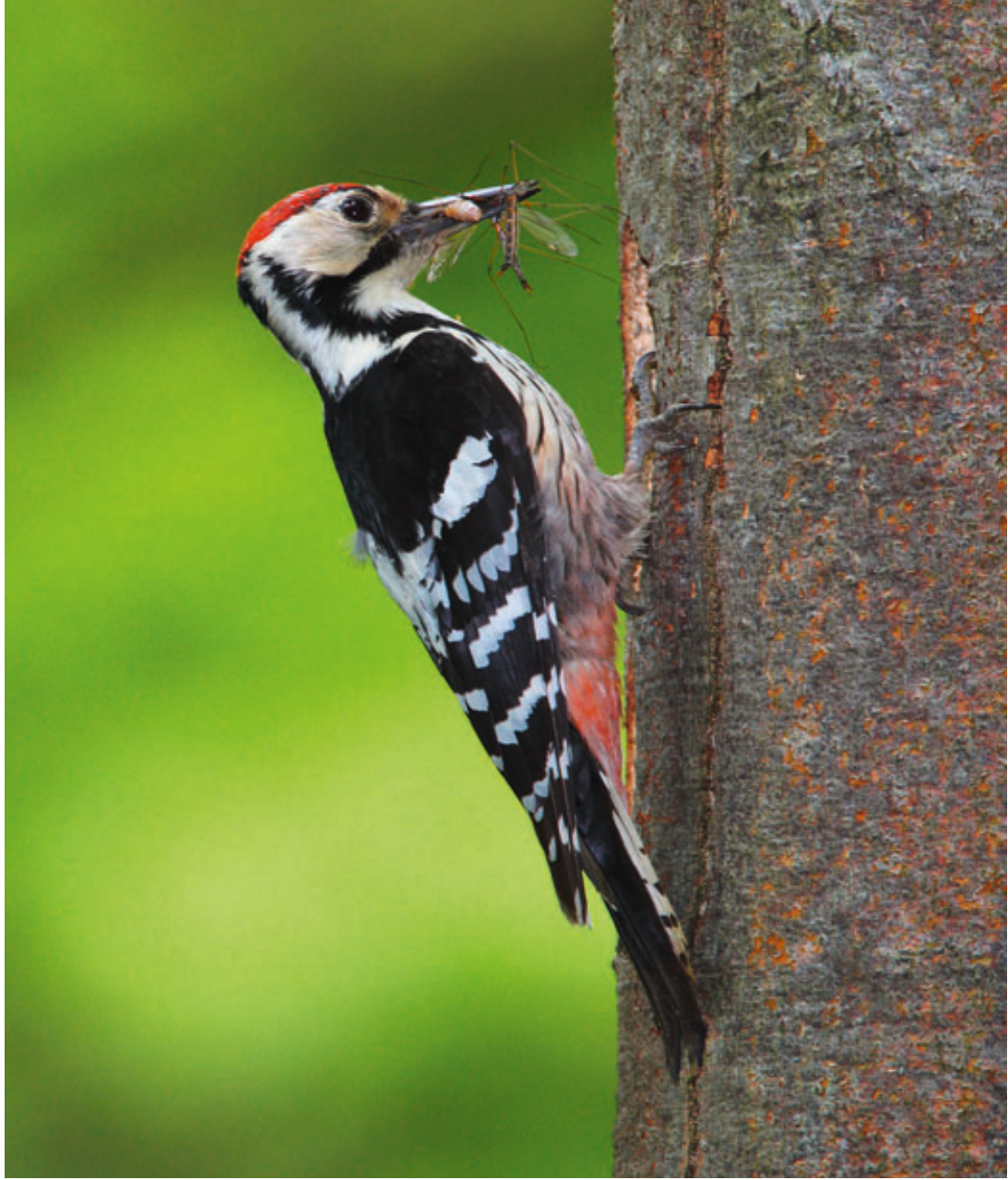

Abb 8 Arten wie der Weissrückenspecht, die nur biologisch alte Wälder mit viel Totholz besiedeln, werden auch Urwald-Reliktarten genannt. Sie eignen sich ausgezeichnet als Zielarten für die Naturwaldreservatsplanung. Foto: Andreas Ebert

Eine weitere Schwierigkeit bei der Planung von Naturwaldreservaten betrifft die Berücksichtigung von trophischen Beziehungsgefügen. Solche können bei den naturräumlichen Gegebenheiten in der mitteleuropäischen Kulturlandschaft nie vollständig in einem Reservat integriert werden. Die saisonale Raumnutzung von Grossherbivoren wie Rothirsch und die Nahrungsökologie des Luchses können in einer Reservatkonzeption nur integriert werden, wenn sie im Verbund und unter Berücksichtigung der Lebensraumverhältnisse ausserhalb der Reservatsgrenzen geplant werden.

Ein grundsätzliches Problem bei der Fokussierung auf Naturwaldreservate bei der Förderung von Altholzspezialisten ist die Zeitverzögerung zwischen einer Lebensraumveränderung und dem regionalen Verschwinden einer Art, das manchmal erst mehrere Generationen später auftritt. Dieses Phänomen ist in der Metapopulationsbiologie als «extinction debt» bekannt (Tilman et al 1994). So kann es sein, dass das Verschwinden von einzelnen Arten trotz Gegenmassnahmen nicht verhindert werden kann, weil die Landschaft als Ganzes ihre Lebensraumkapazität für eine überlebensfähige Population bereits verloren hat. Im Gegenzug können Arten, die als regional ausgestorben gelten, über Jahrzehnte unter der Nachweisschwelle verharren. So wurde zum Bei- spiel der Schnellkäfer Danosoma fasciatus nach über 100 Jahren in einer Totholzfläche in Bayern wieder entdeckt (Müller et al 2008a).

\section{Der Weg zum Ziel}

Aus naturschutzfachlicher Sicht wichtiger als ein absolutes Flächentotal von Naturwaldreservaten ist eine strategische Planung, die sowohl gebietstypische als auch seltene und artenreiche Waldgesellschaften und eine langfristige Sicherung von Altbeständen mit vielfältigen Totholzqualitäten für den Prozessschutz im Wald anstrebt. Bei der Entwicklung eines Verbundsystems von Naturwaldreservaten sollen die Habitatansprüche und Schwellenwerte von wichtigen Zielarten (Prioritäts-, Verantwortungs- und Schirmarten) als Stellvertreter für typische Artengemeinschaften berücksichtigt werden.

Die zentrale Frage auf dem Weg zum Ziel ist, welche Artengruppen berücksichtigt werden sollen. Weil Naturwaldreservate hauptsächlich dem Prozessschutz dienen, eignen sich als Zielarten insbesondere Artengruppen, die an natürliche Störungen sowie Alt- und Totholzstrukturen gebunden sind. Dies sind Arten, die von, auf oder in biologisch alten oder toten Bäumen leben, wie Bockkäfer und xylobionte Vögel, Mollusken, Flechten, Holzpilze und Moose. Es eignen sich auch pyrophile Arten, die oft weit verbreitet sind, aber nur in geringen Dichten vorkommen, dafür aber weite Distanzen überwinden können, um neue Brandflächen zu besiedeln (z.B. die Laufkäfer Pterosticus quadrifoveolatus und Sericoda quadripunctata).

Grundsätzlich ist zu beachten, dass sich Istund Zielzustand in Naturwaldreservaten in den heutigen, häufig einschichtigen Hochwäldern noch stark unterscheiden. Daher ist die Wirkungskontrolle langfristig anzulegen. Allenfalls müssen alternative Vorgehensweisen geprüft werden, zum Beispiel mittels der Einrichtung von Sonderwaldreservaten, in welchen gezielte Eingriffe zur Förderung des Totholzangebots durchgeführt werden, oder mit vorgängigen Massnahmen wie der Entnahme gebietsfremder Baumarten oder dem Rückbau von Entwässerungsgräben, die die Ausgangsbedingungen für Naturwaldreservate verbessern. Naturwaldreservate sind aber unbestritten ein wichtiges Instrument zur Förderung der biologischen Vielfalt. Ob die in der Schweiz und in Deutschland angestrebten 5\% Naturwaldreservate ausreichen, um die auf alte Waldentwicklungsphasen spezialisierten Artengemeinschaften langfristig zu erhalten, ist nicht bekannt. Umso wichtiger ist es, dass bei Reservatsplanungen die oben besprochenen Kriterien berücksichtigt werden, welche die Qualität und damit die naturschutzfachliche Wirkung von Naturwaldreservaten verbessern.

Eingereicht: 14. Juni 2011, akzeptiert (mit Review): 13. März 2012 


\section{Literatur}

AITKEN KEH, WIEBE KL, MARTIN K (2002) Nest-site reuse patterns for a cavity-nesting bird community in interior British Columbia. Auk 119: 391-402.

ALBRECHT L (1988) Ziele und Methoden forstlicher Forschung in Naturwaldreservaten. Schweiz Z Forstwes 139: 373-387.

ALBRECHT L (1990) Grundlagen, Ziele und Methodik der waldökologischen Forschung in Naturwaldreservaten. München: Bayer Staatminist Ernähr, Schr.reihe Naturwaldreservate Bayern 1. 221 p.

ALBRECHT L (1991) Naturwaldreservate - Leitbilder für den Wirtschaftswald. Nationalpark 3: 42-46.

BÄSSLER C, MÜLLER J (2010) Importance of natural disturbance for recovery of the rare polypore Antrodiella citrinella Niemelä \& Ryvarden. Fungal Biol 114: 129-133.

BÄSSLER C, MÜLLER J, DZIOCK F, BRANDL R (2010) Effects of resource availability and climate on the diversity of wooddecaying fungi. J Ecol 98: 822-832.

BERG A ET AL (1994) Threatened plant, animal, and fungus species in Swedish forests - distribution and habitat associations. Conserv Biol 8: 718-731.

BMU (2007) Nationale Strategie zur biologischen Vielfalt. Berlin: Bundesministerium Umwelt Naturschutz Reaktorsicherheit. $178 \mathrm{p}$.

BOBIEC A ET AL (2000) Rich deciduous forests in Białowieża as a dynamic mosaic of developmental phases: premises for nature conservation and restoration management. For Ecol Manage 130: 159-175.

BOLLIGER M, IMESCH N, SCHNIDRIG R (2012) Waldreservatspolitik der Schweiz: Zwischenbilanz und Perspektiven aus Sicht des Bundes (Essay). Schweiz Z Forstwes 163: 199_ 209. doi: 10.3188/szf.2012.0199

BOLLMANN K (2011) Naturnaher Waldbau und Förderung der biologischen Vielfalt im Wald. In: Eidgenöss Forsch.anstalt WSL, editor. Der multifunktionale Wald - Konflikte und Lösungen. Birmensdorf: Eidgenöss Forsch.anstalt Wald Schnee Landschaft, Forum Wissen. pp. 27-36.

BOLLMANN K ET AL (2009) Konzepte, Instrumente und Herausforderungen bei der Förderung der Biodiversität im Wald. Schweiz Z Forstwes 160: 53-67. doi: 10.3188/ szf.2009.0053

BOLLMANN K, GRAF RF, JACOB G, THIEL D (2008) Von der Forschung zur Auerhuhnförderung: eine Projektsynthese. Ornithol Beob 105: 107-116.

BOLLMANN K, GRAF RF, SUTER W (2011) Quantitative predictions for patch occupancy of capercaillie in fragmented habitats. Ecography 34: 276-286.

BOLLMANN K, KELLER V, ZBINDEN N, MÜLLER W (2002) Prioritäre Vogelarten für Artenförderungsprogramme in der Schweiz. Ornithol Beob 99: 301-320.

BONFILS P, BOLLIGER M (2003) Wälder von besonderem genetischem Interesse (BGI-Wälder). Bern: Bundesamt Umwelt Wald Landschaft. $60 \mathrm{p}$

BOUGET C, DUELLI P (2004) The effects of windthrow on forest insect communities: a literature review. Biol Conserv 118: 281-299.

BRANG P, BUGMANN H, BOLLIGER M (2011) Waldreservate in der Schweiz. In: Brang P, Heiri C, Bugmann $\mathrm{H}$, editors. Waldreservate. 50 Jahre natürliche Waldentwicklung in der Schweiz. Bern: Haupt. pp. 26-37.

BROGGI MF, WILLI G (1993) Waldreservate und Naturschutz. Beiträge zum Naturschutz in der Schweiz. Basel: Schweizerischer Bund Naturschutz. $79 \mathrm{p}$.

BÜCKING W (2007) Naturwaldreservate in Europa. Forstarchiv 78: 180-187.
BUGMANN H, BRANG P (2009) Ausgewählte Ergebnisse aus fünfzig Jahren Forschung in Schweizer Naturwaldreservaten. In: Eidgenöss Forsch.anstalt WSL, editor. Langzeitforschung für eine nachhaltige Waldnutzung. Birmensdorf: Eidgenöss Forsch.anstalt Wald Schnee Landschaft, Forum Wissen. pp. 93-102.

BÜHLER U (2009) Totholz - existenziell für den Weissrückenspecht in Nordbünden. Schweiz Z Forstwes 160: 210-217. doi: $10.3188 /$ szf.2009.0210

BUSSLER H, SCHMIDL J (2009) Die xylobionte Käferfauna von sechs Eichen im Naturwaldreservat Eichhall im bayerischen Hochspessart (Coleoptera). Entomol Z 119: 115-123.

BÜTLER R, LACHAT T (2009) Wälder ohne Bewirtschaftung: eine Chance für die saproxylische Biodiversität. Schweiz Z Forstwes 160: 324-333. doi: 10.3188/szf.2009.0324

CARLSON A, SANDSTROM U, OLSSON K (1998) Availability and use of natural tree holes by cavity nesting birds in a Swedish deciduous forest. Ardea 86: 109-119.

CIOLDI F ET AL (2010) Waldressourcen. In: Brändli UB, editor. Schweizerisches Landesforstinventar. Ergebnisse der dritten Erhebung 2004-2006. Eidgenöss Forsch.anstalt Wald Schnee Landschaft. pp. 31-113.

DUC P ET AL (2010) Holzproduktion. In: Brändli UB, editor. Schweizerisches Landesforstinventar. Ergebnisse der dritten Erhebung 2004-2006. Eidgenöss Forsch.anstalt Wald Schnee Landschaft. pp. 143-184.

DUELLI P, WERMELINGER B (2010) Der Alpenbock (Rosalia alpina). Ein seltener Bockkäfer als Flaggschiff-Art. Birmensdorf: Eidgenöss Forsch.anstalt Wald Schnee Landschaft, Merkblatt Praxis 39. 8 p.

EHRBAR R, BOLLMANN K, MOLLET P (2011) Ein Sonderwaldreservat für das Auerhuhn - das Beispiel Amden (Kanton St. Gallen). Schweiz Z Forstwes 162: 11-21. doi: 10.3188/ szf.2011.0011

FENGER M (1996) Implementing biodiversity conservation through the British Columbia forest practices code. For Ecol Manage 85: 67-77.

FRANK G (2002) Brutzeitliche Einnischung des Weissrückenspechts Dendrocopos leucotos im Vergleich zum Buntspecht Dendrocopos major in montanen Mischwäldern der nördlichen Kalkalpen. Vogelwelt 123: 225-239.

FRANK G (2009) Naturwaldreservate in Österreich - von persönlichen Initiativen zu einem systematischen Programm. Mitt Ver Forstl Standortskunde Forstpflanzenzüchtung 46: 23-32.

FRANK G ET AL (2010) Naturwaldreservate in Österreich. Schätze der Natur. Wien: Bundesministerium Land- Forstwirtschaft, Umwelt Wasserwirtschaft. $20 \mathrm{p}$.

GOSSNER MM, MÜLLER J (2011) The influence of species traits and q-metrics on scale-specific b-diversity components of arthropod communities of temperate forests. Landsc Ecol 26: 411-424.

HEIRI C, BRANG P, COMMARMOT B, MATTER JF, BUGMANN H (2011) Walddynamik in Schweizer Naturwaldreservaten: Kennzahlen und Trends. In: Brang P, Heiri C, Bugmann H, editors. Waldreservate. 50 Jahre natürliche Waldentwicklung in der Schweiz. Bern: Haupt. pp. 73-89.

HEIRI C, WOLF A, ROHRER L, BUGMANN H (2009) Forty years of natural dynamics in Swiss beech forests: structure, composition, and the influence of former management. Ecol Appl 19: 1920-1934.

HOF C, LEVINSKY I, ARAUJO MB, RAHBEK C (2011) Rethinking species ability to cope with rapid climate change. Glob Chang Biol 17: 2987-2990.

HOFMANN H, MÜLLER N, SCHNYDER N (2006) Merkblätter Artenschutz - Moose. Bern: Bundesamt Umwelt.24 p. 
ILG C ET AL (2008) Long-term reactions of plants and macroinvertebrates to extreme floods in floodplain grasslands. Ecology 89: 2392-2398.

INDERMÜHLE M, KAUFMANN G, STEIGER P (1998) Konzept Waldreservate Schweiz: Schlussbericht des Projektes Reservatspolitik der Eidgenössischen Forstdirektion. Bern: Bundesamt Umwelt Wald Landschaft. $102 \mathrm{p}$.

IUCN (1994) Guidelines for protected area management categories. Commission on National Parks and Protected Areas (CNPPA). Gland: International Union Conservation Nature. $261 \mathrm{p}$.

KELLER V, BOLLMANN K (2001) Für welche Vogelarten trägt die Schweiz eine besondere Verantwortung? Ornithol Beob 98: 323-340.

KULAKOWSKI D, BEBI P, RIXEN C (2011) The interacting effects of land use change, climate change and suppression of natural disturbances on landscape forest structure in the Swiss Alps. Oikos 120: 216-225.

LANDE R, BARROWCLOUGH G (1987) Effective population size, genetic variation, and their use in population management. In: Soulé M, editor. Viable populations for conservation. Cambridge: Cambridge Univ Press. pp. 87-123.

LEIBUNDGUT H (1962) Urwaldforschung und Waldbau. Allg Forst Z Waldwirtsch Umweltvorsorge 17: 842.

LEIBUNDGUT H (1966) Waldreservate. Schweiz Z Forstw 117: 900-907.

LEIBUNDGUT H (1993) Europäische Urwälder. Wegweiser zur naturnahen Waldwirtschaft. Bern: Haupt. 260 p.

LORENZ J (2010) «Urwaldrelikt»-Käferarten in Sachsen (Coleoptera). Sächsische Entomol Z 5: 59-98.

MARTIKAINEN P, SIITONEN J, KAILA L, PUNTTILA P, RAUH J (1999) Bark beetles (Coleoptera, Scolytidae) and associated beetle species in mature managed and old-growth boreal forests in southern Finland. For Ecol Manage 116: 233-245.

MAYER H, ZUKRIGL K, SCHREMPF W, SCHLAGER G (1987) Urwaldreste, Naturwaldreservate und schützenswerte Naturwälder in Österreich. Wien: Univ Bodenkultur, WaldbauInstitut. $971 \mathrm{p}$.

MEYER P, BÜCKING W, GEHLHAR U, SCHULTE U, STEFFENS R (2007) Das Netz der Naturwaldreservate in Deutschland: Flächenumfang, Repräsentativität und Schutzstatus im Jahr 2007. Forstarchiv 78: 188-196.

MEYER P ET AL (2011) Aufbau eines Systems nutzungsfreier Wälder in Deutschland. Nat Landsch 86: 243-249.

MONING C, MÜLLER J (2009) Critical forest age thresholds for the diversity of lichens, molluscs and birds in beech ( $\mathrm{Fa}$ gus sylvatica L.) dominated forests. Ecol Indic 9: 922-932.

MONING C, BUSSLER H, MÜLLER J (2010) Ökologische Schüsselwerte in Bergmischwäldern als Grundlage für eine nachhaltige Forstwirtschaft. Grafenau: Nationalpark Bayer Wald. $103 \mathrm{p}$.

MORETTI M ET AL (2010) Fire-induced taxonomic and functional changes in saproxylic beetle communities in fire sensitive regions. Ecography 33: 760-771.

MÜLLER J, BUSSLER H, SIMON U, HACKER H (2004) Eichenfurnier trotz Widderbock. Allg Forst Z Waldwirtsch Umweltvorsorge 16: 879-882.

MÜLLER J (2005) Wie beeinflusst Forstwirtschaft die Biodiversität in Wäldern? Eine Analyse anhand der xylobionten Käfer (Insecta: Coleoptera). Beitr bayer Entomofaunistik 7: 1-8.

MÜLLER J ET AL (2005) Urwald relict species - Saproxylic beetles indicating structural qualities and habitat tradition. Waldökologie Online 2: 106-113.

MÜLLER J, GOSSNER M (2007) Single host trees in a closed forest canopy matrix: a highly fragmented landscape. J Appl Entomol 131: 613-620.
MÜLLER J, ENGEL H, BLASCHKE M (2007A) Assemblages of wood-inhabiting fungi related to silvicultural management intensity in beech forests in southern Germany. Eur J For Res 126: 513-527.

MÜLLER J, HOTHORN T, PRETZSCH H (2007B) Long-term effects of logging intensity on structures, birds, saproxylic beetles and wood-inhabiting fungi in stands of European beech Fagus sylvatica L. For Ecol Manage 242: 297-305.

MÜLLER J, BUSSLER H, GOSSNER M, RETTELBACH T, DUELLI P (2008A) The European spruce bark beetle Ips typographus in a national park: from pest to keystone species. Biodivers Conserv 17: 2979-3001.

MÜLLER J, BUSSLER H, KNEIB T (2008B) Saproxylic beetle assemblages related to silvicultural management intensity and stand structures in a beech forest in Southern Germany. J Insect Conserv 12: 107-124.

MÜLLER J, GOSSNER M (2010) Three-dimensional partitioning of diversity reveals baseline information for state-wide strategies for the conservation of saproxylic beetles. Biol Conserv 143: 625-633.

MÜLLER J, REED N, BUSSLER H, BRANDL R (2010A) Learning from a "benign neglect strategy" in a national park: Response of saproxylic beetles to dead wood accumulation. Biol Conserv 143: 2559-2569.

MÜLLER J, STADLER J, BRANDL R (2010B) Composition versus physiognomy of vegetation as predictors of bird assemblages: the role of lidar. Remote Sens Environ 114: 490-495.

MÜLLER M, LACHAT T, BÜTLER R (2012) Wie gross sollen Altholzinseln sein? Schweiz Z Forstwes 163: 49-56. doi: 10.3188/ szf. 2012.0049

PAILLET Y ET AL (2010) Biodiversity differences between managed and unmanaged forests: Meta-analysis of species richness in Europe. Conserv Biol 24: 101-112.

PALTTO H, NORDEN B, GOTMARK F, FRANC N (2006) At which spatial and temporal scales does landscape context affect local density of Red Data Book and Indicator species? Biol Conserv 133: 442-454.

PRADELLA C, WERMELINGER B, OBRIST M, DUELLI P, MORETTI M (2010) On the occurrence of five pyrophilous beetle species in the Swiss Central Alps (Leuk, Canton Valais). Mitt Schweiz Entomol Ges 83: 187-197.

PRESSEY RL, CABEZA M, WATTS ME, COWLING RM, WILSON KA (2007) Conservation planning in a changing world. Trends Ecol Evol 22: 583-592.

PRO NATURA (2011) Schweizer Wald: Ein Alleskönner steht unter Druck. Basel: Pro Natura. 48 p.

RAABE S ET AL (2010) Drivers of bryophyte diversity allow implications for forest management with a focus on climate change. For Ecol Manage 260: 1956-1964.

RANIUS T (2006) Measuring the dispersal of saproxylic insects: a key characteristic for their conservation. Popul Ecol 48: 177-188.

REICH T, LÄSSIG R, WOHLGEMUTH T (2010) Waldentwicklung nach Windwurf im Waldreservat Rorwald, Kanton Obwalden. Birmensdorf: Eidgenöss Forsch.anstalt Wald Schnee Landschaft. $44 \mathrm{p}$.

SCHERRER D, KÖRNER C (2011) Topographically controlled thermal-habitat differentiation buffers alpine plant diversity against climate warming. J Biogeogr 38: 406-416.

SCHERZINGER W (1996) Naturschutz im Wald: Qualitätsziele einer dynamischen Waldentwicklung. Stuttgart: Ulmer. $447 \mathrm{p}$.

SCHIEGG K (2000A) Effects of dead wood volume and connectivity on saproxylic insect species diversity. Ecoscience 7: 290-298. 
SCHIEGG K (2000B) Are there saproxylic beetle species characteristic of high dead wood connectivity? Ecography 23: 579-587.

SCHIESS H, SCHIESS-BÜHLER C (1997) Dominanzminderung als ökologisches Prinzip: die Neubewertung der ursprünglichen Waldnutzung für den Arten- und Biotopschutz am Beispiel der Tagfalterfauna eines Auenwaldes in der Nordostschweiz. Mitt Eidgenöss Forsch.anst Wald Schnee Landsch 72: 127.

SEIBERT P, HAGEN J (1974) Zur Auswahl von Waldreservaten in Bayern. Forstwiss Centbl 93: 274-284.

SIITONEN J (2001) Forest management, coarse woody debris and saproxylic organisms: Fennoscandian boreal forests as example. Ecol Bull 49: 11-41.

SIITONEN J, SAARISTO L (2000) Habitat requirements and conservation of Pytho kolwensis, a beetle species of old-growth boreal forest. Biol Conserv 94: 211-220.

SVS (2011) Biodiversität: Vielfalt im Wald. Zürich: Schweiz Vogelschutz. 39 p.

TILMAN D, MAY RM, LEHMAN CL, NOWAK MA (1994) Habitat destruction and the extinction debt. Nature 371: 65-66.

USBECK T ET AL (2010) Increasing storm damage to forests in Switzerland from 1858 to 2007. Agric For Meteorol 150: 47-55.

WALENTOWSKI H ET AL (2010) Sind die deutschen Waldnaturschutzkonzepte adäquat für die Erhaltung der buchenwaldtypischen Flora und Fauna? Eine kritische Bewertung basierend auf der Herkunft der Waldarten des mitteleuropäischen Tief- und Hügellandes. Forstarchiv 81: 195-217.

\section{Naturwaldreservate: welche, wo und wofür? (Essay)}

Welche Fläche braucht es für Naturwaldreservate in Mitteleuropa? Interessanterweise steht diese Frage im Zentrum der aktuellen Debatte über den Bedarf an ungenutzten Wäldern für den Biodiversitätsschutz. Dabei dürften in einem System mit naturnahem Waldbau die Qualität, Lage, Zusammensetzung und Verteilung von Naturwaldreservaten entscheidender sein, wenn es darum geht, die Vielfalt an Arten, Lebensgemeinschaften und natürlichen Prozessen im Wald zu fördern. Streng genommen kann die Frage nach dem minimal notwendigen Flächenanteil nur im Zusammenhang mit den übergeordneten Naturschutzzielen beantwortet werden. Zudem wird der erforderliche Flächenanteil von anderen $\mathrm{Na}$ turvorrangflächen wie Sonderwaldreservaten, Altholzinseln und sonstigen Wäldern von besonderer naturschutzfachlicher Bedeutung sowie den generellen Anforderungen an den naturnahen Waldbau beeinflusst. Weil konkrete, übergeordnete Ziele für die Waldbiodiversität in Mitteleuropa weitgehend fehlen, legen wir den Schwerpunkt dieses Artikels auf die Kriterien, welche die naturschutzfachliche Wirkung von Naturwaldreservaten positiv beeinflussen. Solche sind u.a. die Grösse und Kompaktheit eines Reservats, seine Habitatkontinuität und Vernetzung, die Repräsentativität der Waldtypen, ihre Artenausstattung und biogeografische Verteilung, die standörtliche Vielfalt und Naturnähe der Vegetation sowie die Abundanz von Schlüsselstrukturen und Zielarten.
WARREN MS, KEY RS (1991) Woodlands: past, present and potential for insects. In: Collins NM, Thomas JA, editors. The conservation of insects and their habitats. London: Academic Press. pp. 155-212.

WELZHOLZ JC, JOHANN E (2007) History of protected forest areas in Europe. In: Frank $G$ et al, editors. Protected forest areas in Europe - analysis and harmonisation (PROFOR): Results, conclusions and recommendations. Vienna: Federal Research Training Centre Forests Natural Hazards Landscape. pp. 17-40.

WERMELINGER B (2004) Ecology and management of the spruce bark beetle Ips typographus - a review of recent research. For Ecol Manage 202: 67-82.

WERMELINGER B, DUELLI P, OBRIST M, ODERMATT O, SEIFERT M (1995) Die Entwicklung der Fauna auf Windwurfflächen mit und ohne Holznutzung. Schweiz Z Forstwes 146: 913-928.

WINTER S, FLADE M, SCHUMACHER H, KERSTAN E, MÖLLER G (2005) The importance of near natural stand structures for the biocoenosis of lowland beech forests. For Snow Landsc Res 79: 127-144.

WINTER S, MÖLLER G (2008) Microhabitats in lowland beech forests as monitoring tool for nature conservation. For Ecol Manage 255: 1251-1261.

WOHLGEMUTH TET AL (2010A) Leben mit Waldbrand. Birmensdorf: Eidgenöss Forsch.anstalt Wald Schnee Landschaft, Merkblatt für die Praxis. $16 \mathrm{p}$.

WOHLGEMUTH T, HESTER C, JOST AR, WASEM U, MOSER B (2010B) Dynamik der Wiederbewaldung im Waldbrandgebiet von Leuk (Wallis). Schweiz Z Forstwes 161: 450-459. doi: 10.3188/szf.2010.0450

\section{Les réserves forestières naturelles: critères de sélection, où et moyennant quoi? (Essai)}

La question: «Quelle est la superficie nécessaire pour les réserves forestières naturelles en Europe centrale?» est au cœur du débat actuel sur la nécessité de forêts non aménagées pour la protection de la biodiversité. A l'intérieur d'une gestion forestière proche de la nature, la qualité, l'emplacement, la composition et la distribution de réserves forestières naturelles pourraient avoir des impacts plus significatifs sur la diversité des espèces, les communautés et les processus naturels que la superficie des réserves seule. Strictement parlant, la question de la superficie minimale nécessaire des réserves ne peut être répondue qu'en fonction des objectifs de conservation généraux. En outre, le ratio requis de réserves naturelles forestières par rapport à la superficie forestière totale est influencé par d'autres facteurs comme l'abondance et la distribution de réserves forestières spéciales, de biotopes forestiers protégés et d'îlots de vieux peuplements forestiers, ainsi que les normes générales pour une sylviculture proche de la nature. Parce que des objectifs concrets et globaux pour la protection de la biodiversité des forêts en Europe centrale sont manquants, nous avons mis l'accent dans cet article sur les critères qui favorisent l'impact positif des réserves forestières naturelles sur la biodiversité. Ce sont entre autres la taille et la compacité d'une réserve, sa continuité de l'habitat et la connectivité, la représentativité des types forestiers, leur composition en espèces et leur distribution biogéographique, l'hétérogénéité du site et le caractère naturel de la végétation et l'abondance des structures clés et des espèces cibles. 